\title{
Conduite et complications de l'oxygénation extracorporelle veinoveineuse
}

\author{
Management and Complications of Venovenous Extracorporeal Membrane Oxygenation
}

\author{
L.-M. Jacquet \\ (C) SRLF et Springer-Verlag France 2014
}

\begin{abstract}
Résumé Cet article se propose de tracer, à la lumière de la littérature, les grandes lignes de la prise en charge des patients traités par oxygénation extracorporelle veinoveineuse pour une insuffisance respiratoire réfractaire aux traitements conventionnels. Nous diviserons la période d'assistance externe en trois phases successives : la phase d'initiation du traitement, une phase d'entretien et une phase de sevrage et d'arrêt de la thérapeutique. À chacune de ces périodes, nous nous attacherons à décrire les réglages et la surveillance du circuit extérieur ainsi que le traitement et la surveillance du patient lui-même. Nous nous attacherons également à décrire à chaque étape les complications le plus fréquemment rencontrées et les traitements proposés.
\end{abstract}

\section{Mots clés ECMO - Complication · Surveillance}

\begin{abstract}
This article reviews the management of patients treated with venovenous extracorporeal membrane oxygenation $(E C M O)$ for acute respiratory failure refractory to the conventional therapies. The period of extracorporeal respiratory support can be divided in three successive periods: the period of ECMO initiation, the period of treatment with ECMO, and the period of ECMO weaning. We will describe the main technical aspects of ECMO as well as the monitoring of the extracorporeal circuit and the ECMO-treated patient. The most frequent complications in each period of the management of ECMO-treated patients will be described and the possible adequate solutions will be considered.
\end{abstract}

Keywords $\mathrm{ECMO} \cdot$ Complication $\cdot$ Monitoring

\footnotetext{
L.-M. Jacquet ( $\square$ )

Service de pathologies cardiovasculaires intensives, Cliniques universitaires Saint-Luc, avenue Hippocrate-10, B-1200 Bruxelles, Belgique

e-mail : luc-marie.jacquet@uclouvain.be
}

\section{Initiation du traitement}

Le traitement de l'insuffisance respiratoire du syndrome de détresse respiratoire aiguë (SDRA) par échanges gazeux extracorporels fait le plus souvent appel à une technique veinoveineuse d'oxygénation extracorporelle par membrane (ECMO VV). Comme décrit antérieurement, plusieurs formes de canulation sont possibles, le drainage par une longue canule en veine fémorale avec retour par une plus courte canule en veine jugulaire étant la technique la plus fréquemment utilisée chez l'adulte. L'utilisation d'une canule unique à double lumière placée en veine jugulaire interne sous contrôle radioscopique ou échocardiographique est, cependant, en augmentation constante depuis 2009 [1].

$\mathrm{Au}$ début du traitement, le débit du circuit est réglé pour être le plus proche possible du débit cardiaque du patient. En effet, le sang qui arrive dans l'artère pulmonaire est un mélange du sang qui a traversé le circuit extracorporel et du sang veineux désaturé qui n'a pas circulé en dehors de l'organisme. Considérant la gravité habituelle de l'atteinte pulmonaire et la volonté de réduire au maximum l'agressivité de la ventilation mécanique, la contribution des poumons à l'oxygénation sanguine est généralement très faible, et la saturation artérielle systémique est fort proche de la saturation dans l'artère pulmonaire. L'oxygénation du sang du circuit externe dépend essentiellement de la fraction d'oxygène dans le gaz délivré à l'oxygénateur membranaire $\left(\mathrm{F}_{\mathrm{d}} \mathrm{O}_{2}\right)$ qui sera de 1 au départ permettant de saturer l'hémoglobine à $100 \%$ (pour autant que le débit sanguin à travers l'oxygénateur soit inférieur au débit nominal de l'oxygénateur choisi). La saturation en oxygène du sang de l'artère pulmonaire sera dès lors fonction du rapport entre le débit du circuit, dont l'hémoglobine est saturée à $100 \%$, et le débit veineux résiduel, dont la saturation devrait être de l'ordre de $70 \%$. Comme le débit cardiaque est la somme de ces deux débits, plus le rapport entre le débit du circuit et le débit cardiaque sera élevé, plus la saturation dans l'artère 
pulmonaire et la saturation artérielle systémique seront hautes. Il est dès lors primordial de pouvoir délivrer un débit extracorporel proche du débit cardiaque du patient en choisissant une vitesse de rotation de la pompe adéquate.

La plupart des pompes utilisées pour le support extracorporel de patients adultes étant des pompes centrifuges, il faudra toutefois éviter des vitesses de rotation trop élevées qui peuvent engendrer des pressions d'aspiration trop négatives et/ou d'éjection trop élevées. Lorsque la ligne de drainage est occluse, la pression d'aspiration ne devrait pas descendre en dessous de $-300 \mathrm{mmHg}$ [2]. À ce niveau de pression, qui peut survenir en cas de succion (l'aspiration trop importante par rapport au retour veineux se manifestant par des tremblements de la ligne de drainage), des phénomènes de cavitation peuvent s'observer. Sous l'effet de la dépression, les gaz dissous peuvent passer en phase gazeuse, et l'interface gaz/sang ainsi créée est un puissant agent inducteur d'hémolyse [3]. Des pressions d'éjection élevées, au-delà de $400 \mathrm{mmHg}$, peuvent également être responsables d'épisodes hémolytiques suite aux forces de cisaillement élevées ainsi crées, sans compter le risque de rupture des lignes ou des connexions que de telles pressions font courir. Rappelons ici qu'un débitmètre doit être placé et calibré sur le circuit pour en mesurer le débit exact qui ne peut être estimé à partir de la vitesse de rotation.

Les saturations artérielles visées en début de traitement seront donc relativement faibles. Les recommandations de l'Extracorporeal Life Support Organization (ELSO) sont de tendre vers une saturation artérielle de $80 \%$ et une saturation veineuse de $70 \%$ [2]. La plupart des équipes visent une saturation artérielle supérieure à $88 \%$, mais il est vrai que dans des situations de haut débit cardiaque qu'on peut rencontrer dans les états septiques ou toxiques, il faut parfois se contenter de saturations bien inférieures. Lorsqu'il n'est pas possible d'atteindre une oxygénation acceptable, il est parfois nécessaire d'ajouter une canule de drainage supplémentaire pour pouvoir augmenter le débit extérieur.

Un élément important dans l'évaluation du débit et de l'efficience du circuit est de tenir compte de la recirculation, c'est-à-dire de la quantité de sang oxygéné et décarboxylé envoyé par la canule de réinjection qui est immédiatement réaspiré par la canule de drainage et qui ne circule donc pas dans l'organisme. La quantité de sang qui recircule doit être soustraite du débit du circuit pour quantifier le débit réellement efficace [4]. Il sera donc recommandé de contrôler rapidement par radiographie le positionnement correct des deux canules et la distance entre les deux extrémités. La recirculation peut être calculée en comparant la saturation du sang veineux, prélevé, par exemple, par une voie centrale en amont de la canule de retour, avec la saturation du sang dans la branche de drainage du circuit. L'utilisation d'une seule canule à double lumière drainant le sang dans les deux veines caves pour le réinjecter en face de l'orifice de la valve tricuspide est, à ce point de vue, particulièrement intéressante même si la mise en place est un peu plus complexe $[5,6]$.

L'élimination du $\mathrm{CO}_{2}$ est beaucoup moins dépendante du débit du circuit, tant l'efficacité des oxygénateurs membranaires est élevée pour l'extraction de ce gaz. Avec des débits sanguins de 1 à $21 / \mathrm{min}$, il est possible d'éliminer pratiquement la totalité du $\mathrm{CO}_{2}$ produit par minute. La quantité de $\mathrm{CO}_{2}$ éliminée est déterminée par le débit de gaz frais qui entre dans l'oxygénateur (communément appelé balayage) qui est réglé au niveau du mélangeur de gaz. Au départ, puisque le débit sanguin dans le circuit est élevé pour maintenir l'oxygénation, il est recommandé de choisir un flux de balayage égal au débit sanguin extracorporel puis d'adapter le balayage en fonction de la $\mathrm{PaCO}_{2}$. En cas d'hypercapnie préexistante, la correction peut être très rapide, mais il est prudent de viser une correction progressive pour éviter le développement d'une alcalose et de modifications de la perfusion cérébrale [2].

C'est en profitant de la haute efficacité des membranes à éliminer le $\mathrm{CO}_{2}$ que se sont développées des techniques d'épuration du dioxyde de carbone artérioveineuse ou veinoveineuse à bas débit. Nous y reviendrons plus loin.

Pour surveiller l'efficacité de l'oxygénateur membranaire, il sera nécessaire de faire des prélèvements sanguins chez le patient ainsi que sur les branches de drainage et de réinjection du circuit. Il est possible sur certains circuits de suivre en continu les saturations avant et après la membrane par oxymétrie de transmission. De cette façon, l'adéquation du transport d'oxygène par rapport aux besoins, reflétée par la saturation veineuse, peut être monitorisée en permanence de même que l'efficience de l'oxygénateur. Le nombre de prélèvements pourra dès lors être réduit. $\mathrm{La} \mathrm{PaCO}_{2}$ sera suivie sur les prélèvements artériels du patient et ne devra être vérifiée sur le circuit qu'en cas de modification inexpliquée. Lors des prélèvements sur le circuit, principalement sur la branche de drainage, une prudence extrême est requise pour éviter toute entrée d'air dans le circuit qui pourrait avoir des conséquences désastreuses.

Toujours au niveau du circuit, on s'assurera de la connexion correcte et du réglage adéquat de l'échangeur thermique. Une proportion significative du sang circule en dehors de l'organisme, et des dérèglements thermiques rapides sont possibles si, par exemple, le circulateur entre l'échangeur thermique et l'oxygénateur ne fonctionne pas correctement. Les principaux éléments du circuit à surveiller ainsi que les complications rencontrées avec ces composants sont repris dans la Tableau 1.

La conduite de la ventilation doit principalement permettre de limiter au maximum les effets secondaires de la ventilation mécanique. Quel que soit le mode de ventilation 


\begin{tabular}{|c|c|c|c|}
\hline Composant & Paramètre à suivre & Moyen de surveillance & Complication \\
\hline Pompe & $\begin{array}{l}\text { Vitesse de rotation } \\
\text { Débit }\end{array}$ & $\begin{array}{l}\text { Déterminée par l'opérateur } \\
\text { Mesuré par débitmètre/alarmes à régler }\end{array}$ & $\begin{array}{l}\text { Thrombose } \\
\text { Hémolyse } \\
\text { Arrêt inopiné }\end{array}$ \\
\hline Oxygénateur & $\begin{array}{l}\text { Efficacité } \\
\text { Fraction délivrée d'oxygène } \\
\text { Gaz de balayage }\end{array}$ & $\begin{array}{l}\mathrm{Si} \text { disponible, oxymétrie continue } \mathrm{S}_{\mathrm{v}} \mathrm{O}_{2} \text { et } \mathrm{S}_{\mathrm{a}} \mathrm{O}_{2} \\
\text { Prélèvements répétés pour analyse des gaz } \\
\text { - à adapter selon } \mathrm{S}_{\mathrm{a}} \mathrm{O}_{2} \\
\text { - à adapter selon } \mathrm{PaCO}_{2}\end{array}$ & $\begin{array}{l}\text { Perte d'efficience } \\
\text { - thrombose } \\
\text { - rupture de fibres } \\
\text { Entrée d'air }\end{array}$ \\
\hline Tubulure du circuit & Pression & $\begin{array}{l}\text { Mesure des pressions de drainage et de retour } \\
\text { Pression préoxygénateur } \\
\text { Alarmes à régler }\end{array}$ & $\begin{array}{l}\text { Rupture de tubulure } \\
\text { Thrombose } \\
\text { Hémolyse si pressions } \\
\text { élevées }\end{array}$ \\
\hline Échangeur thermique & Température & Vérifier température souhaitée et réelle & Arrêt de circulation \\
\hline Canules & $\begin{array}{l}\text { Fixation correcte } \\
\text { Signes d'infection }\end{array}$ & $\begin{array}{l}\text { Au moins } 2 \text { sutures } \\
\text { Rougeur, suintement }\end{array}$ & $\begin{array}{l}\text { Mobilisation de canule } \\
\text { Septicémies }\end{array}$ \\
\hline
\end{tabular}

choisi, il faudra limiter la pression inspiratoire maximale à $25 \mathrm{cmH}_{2} \mathrm{O}$, le volume courant à $6 \mathrm{ml} / \mathrm{kg}$ de poids idéal en maintenant une pression positive de fin d'expiration à 5-15 $\mathrm{cmH}_{2} \mathrm{O}$. La fréquence respiratoire pourra être diminuée à moins de dix ventilations par minute, tandis que la $\mathrm{F}_{\mathrm{i}} \mathrm{O}_{2}$ sera réduite progressivement en dessous de 0,6. La question reste débattue de savoir si une ventilation encore moins agressive en utilisant des volumes inférieurs à $3 \mathrm{ml} / \mathrm{kg}$, par exemple, ou des fréquences respiratoires de quatre à six par minute devrait être recommandée pour promouvoir la guérison pulmonaire [7,8].

Durant la mise en place du circuit, il est souvent nécessaire de placer le patient sous sédation et de maintenir une sédation dans les heures qui suivent. Parfois, il est même nécessaire d'ajouter une curarisation pour assurer l'adaptation du patient au respirateur, mais l'administration de curares n'est à envisager qu'en cas de désaturation artérielle sous $85 \%$.

La mise en contact du sang avec le matériel étranger du circuit nécessite la mise en route d'une anticoagulation systémique. Une dose de charge d'héparine est administrée lors de la mise en place des canules, et une perfusion continue doit être poursuivie en visant un allongement du temps de coagulation du sang total à 180-200 secondes et/ou du temps de thromboplastine partiel activé à 1,5-2 fois la limite supérieure de la normale. Les doses d'héparine nécessaires varient grandement en fonction de l'état du patient (thrombopénie, insuffisance rénale, état hypercoagulable...), de sorte qu'une dose standard est impossible à déterminer. Puisque l'héparine agit par l'intermédiaire de l'activation de l'antithrombine-III plasmatique, des dosages de cette molécule sont utiles. Un déficit en antithrombine-III doit être suspecté si l'anticoagulation souhaitée n'est pas atteinte ou si des signes de coagulation du circuit apparaissent malgré de hautes doses d'héparine. Il peut être utile d'administrer du plasma frais congelé, voire des concentrés d'antithrombine-III dans ces situations $[9,10]$.

Les circuits couverts soit d'héparine, soit d'autres agents chimiques (phosphorylcholine, poly-2-méthoxyéthyl acrylate, etc.) semblent diminuer l'intensité des interactions sang/ biomatériaux, et ils permettent, si des saignements importants apparaissent, de diminuer, voire d'arrêter l'anticoagulation systémique avec des risques limités de formation de thrombus pour autant que le débit dans le circuit ne soit pas trop faible $[11,12]$.

À moins d'avoir eu le temps de remplir le circuit avec du sang, il faut s'attendre à une chute significative de l'hématocrite suite à l'hémodilution par le liquide de remplissage et, donc, à la nécessité de transfuser le patient. Les recommandations de l'ELSO sont de transfuser des globules rouges pour maintenir un hématocrite normal. Si la saturation artérielle est acceptable ( $>88 \%$ ) et que le débit cardiaque est normal, voire élevé, des taux d'hémoglobine inférieurs sont tolérables pour autant que le transport d'oxygène reste adéquat [13].

Puisque l'hémodynamique n'est que peu influencée par la mise en route du circuit externe, la prise en charge hémodynamique suivra les directives généralement appliquées en réanimation. Du fait de l'augmentation du volume sanguin circulant et de l'adsorption possible de certaines molécules pharmacologiques sur les polymères du circuit, il peut être nécessaire d'adapter les doses de certaines médications administrées pour en maintenir l'effet thérapeutique souhaité $[14,15]$. 


\section{Phase de maintenance}

Le débit du circuit d'assistance sera adapté pour obtenir la saturation artérielle en oxygène souhaitée tout en maintenant une ventilation non agressive et des pressions d'aspiration et d'injection sur le circuit dans les limites décrites plus haut.

Dès que l'état clinique le permet, la sédation sera diminuée, l'idéal étant d'avoir un patient réveillé mais légèrement sédaté pour éviter des mouvements intempestifs qui pourraient provoquer une mobilisation des canules et les complications qui ne manqueraient pas de se manifester. Les efforts de toux importants et répétés peuvent parfois empêcher un drainage correct vers le circuit dont le débit ne peut alors plus être maintenu, ce qui nécessite parfois de garder une sédation plus profonde que souhaité. Différents modes de ventilation sont utilisés (ventilation contrôlée, pression positive continue des voies aériennes, ventilation avec relâchement de la pression des voies aériennes...). Aucune étude n'a comparé les modes ventilatoires au cours de l'ECMO, et la seule recommandation qui peut être faite est de limiter les pressions, les volumes et la fraction inspirée d'oxygène pour ne pas léser davantage les poumons.

La saturation artérielle en oxygène de même que les saturations des branches de drainage et de réinjection du circuit continueront à être monitorisées pour s'assurer que les objectifs visés sont atteints et que l'oxygénateur membranaire fonctionne correctement. Des prélèvements pour analyse des gaz sanguins seront effectués au besoin, mais au minimum une fois par jour. Une chute de pression partielle en oxygène à moins de $150 \mathrm{mmHg}$ après l'oxygénateur avec une fraction d'oxygène délivré égale à 1 nécessite le remplacement de l'oxygénateur. Les oxygénateurs récents sont conçus pour être utilisés plusieurs semaines, et il est devenu rarement nécessaire de changer l'oxygénateur sauf pour des supports de durée très prolongée ou en cas d'anticoagulation nettement insuffisante. La perte d'efficience est, en effet, très souvent causée par l'obstruction des fibres par des caillots en formation.

Les paramètres de coagulation seront régulièrement évalués et particulièrement le temps de coagulation et/ou le temps de thromboplastine partiel activé pour ajuster les doses d'héparine. La numération des plaquettes sanguines sera suivie de près, car une thrombopénie apparaît quasi systématiquement suite à l'activation des plaquettes au contact du matériel étranger. Les plaquettes activées ont tendance à former des microaggrégats qui sont captés par le système réticuloendothélial du foie et de la rate. L'anticoagulation nécessaire associée à la consommation de plaquettes et de facteurs de coagulation dans le circuit expliquent la fréquence des complications hémorragiques rencontrées. Récemment, la survenue d'un syndrome de von Willebrand acquis a été mise en évidence dans une série de
31 patients sur 32 sous support extracorporel. Les forces de cisaillement élevées dans la pompe pourraient favoriser le déplissement des multimères de haut poids moléculaire $\mathrm{du}$ facteur von Willebrand et favoriser leur destruction par la protéine ADAMST-13. La disparition des multimères de haut poids moléculaire diminuerait les interactions entre les plaquettes et l'endothélium lésé, ce qui pourrait favoriser la survenue d'accidents hémorragiques [16]. Selon les définitions et les séries, l'incidence des hémorragies oscille entre 15 et $54 \%$ des cas $[1,17,18]$. Elles peuvent survenir en de nombreuses localisations, mais les plus dévastatrices sont les saignements intracrâniens qui surviennent chez 9 à $12 \%$ des patients et sont associés à une augmentation significative de la mortalité $[18,19]$. Les sites de canulation sont une source fréquente d'hémorragie avec des incidences rapportées entre 4 et $20 \%$. On relèvera également la survenue fréquente de saignements du tractus respiratoire supérieur (10 à $13 \%$ ) et du tube digestif (3 à $10 \%$ ). En cas de saignement significatif, l'anticoagulation peut être diminuée et même totalement arrêtée le temps de contrôler la situation. La diminution de l'anticoagulation sera plus sécure si le circuit est couvert d'héparine ou possède une surface traitée. Des globules rouges seront transfusés pour maintenir l'hématocrite proche de la normale. Du plasma frais congelé sera administré en cas d'anomalie de l'hémostase (International Normalized Ratio [INR] $>1,5$ ), et du fibrinogène sera donné si la fibrinogénémie est inférieure à $100 \mathrm{mg} / \mathrm{dl}$. Il faudra souvent transfuser des plaquettes pour maintenir leur taux à plus de $100000 / \mu 1$. Même si le nombre de plaquettes n'est pas effondré, il existe souvent une thrombopathie qui peut être mise en évidence par des tests de réactivité plaquettaire (multiplate...). Des agents antifibrinolytiques (acide tranexamique, acide aminocaproïque) peuvent aider à contrôler un saignement surtout au niveau de sites chirurgicaux. Le facteur VII recombinant activé a quelquefois été utilisé avec succès pour tarir un saignement grave, mais le risque thromboembolique n'est pas négligeable.

L'utilisation d'héparine non fractionnée peut entraîner la synthèse d'anticorps dirigés contre le complexe héparinefacteur 4 plaquettaire. Ces anticorps se lient aux plaquettes entraînant leur activation et leur consommation (thrombopénie induite par l'héparine ou TIH). Une diminution soudaine du nombre de plaquettes doit pousser à réaliser les tests immunologiques et/ou fonctionnels plaquettaires pour confirmer le diagnostic. La survenue d'une TIH, qui compliquerait 5 à $10 \%$ des cas, nécessite l'arrêt de l'administration d'héparine et l'utilisation d'autres anticoagulants (argatroban, lépirudine, bivalirudine...) [20,21]. Des données contradictoires ont été publiées concernant l'utilisation de circuits couverts d'héparine dans ces circonstances, mais il nous paraît plus prudent d'éliminer également ces circuits qui pourraient entretenir la réaction immunologique $[21,22]$. 
Des complications thrombotiques peuvent survenir suite à l'incapacité de moduler l'activation de la coagulation malgré le traitement anticoagulant. L'apparition de caillots dans le circuit est rapportée dans $20 \%$ des cas et est favorisée par une anticoagulation insuffisante et/ou un bas débit dans le circuit. Des caillots peuvent se former à tout endroit du circuit, mais ils apparaissent le plus souvent du côté veineux (en amont de l'oxygénateur membranaire), dans l'oxygénateur ou dans la tête de pompe. Le composant atteint doit être remplacé, et il est parfois nécessaire de remplacer tout le circuit. Même s'ils ne sont pas visibles, les caillots dans le circuit entraînent fréquemment une hémolyse significative.

Le changement du circuit ou d'un de ses composants peut se faire rapidement lorsque le personnel est formé et entrainé. Il faudra toutefois anticiper l'arrêt de la circulation externe et majorer le support ventilatoire avant de clamper les lignes du circuit. Un double clampage est recommandé, un clamp étant placé près du patient sur chacune des lignes, et un deuxième clamp est fixé de part et d'autre du composant à changer. Il faut bien sûr être très attentif et rigoureux pour éviter toute pénétration d'air dans le circuit, surtout du côté de la réinjection.

Des désordres plus profonds de la coagulation sont décrits chez 1-2\% des patients, en particulier, une coagulation intravasculaire disséminée responsable d'une consommation de tous les facteurs de coagulation, du fibrinogène et des plaquettes.

Une hémolyse éventuelle doit être recherchée activement par le dosage de l'hémoglobine plasmatique qui est normalement inférieur à $10 \mathrm{mg} / \mathrm{dl}$. Une élévation des lacticodéshydrogénases (LDH), de la bilirubine non conjuguée et des schizocytes de même que la coloration des urines sont également évocateurs d'une destruction trop importante des hématies. L'hémolyse peut être liée à des forces de cisaillement trop élevées si la pression d'aspiration est trop négative, ce qui peut se produire si l'aspiration de la pompe dépasse le retour veineux. Si les pressions d'éjection sont trop élevées parce que la canule de réinjection offre une résistance trop importante (canule trop étroite pour le débit souhaité ou caillot en formation dans la canule), les forces de cisaillement créées peuvent aussi être responsables d'une hémolyse. Très fréquemment, la destruction des globules rouges est due à la formation de caillots dans la tête de pompe et particulièrement au niveau de l'axe de support du rotor. Ce risque est évidemment moindre pour les pompes dépourvues d'axe mécanique dont le maintien du rotor est assuré par une suspension magnétique.

Même si la ventilation mécanique est maintenue à un niveau d'agression minimal, il n'est pas rare de voir apparaître des pneumothorax que l'on pourrait presque qualifier de spontanés. Un pneumothorax de petite taille $(<20 \%$ du champ pulmonaire) ne doit pas nécessairement être drainé et peut se résorber spontanément. Les pneumothorax de plus de $20 \%$ de la surface pulmonaire devront par contre être drainés mais avec la plus grande prudence, car des hémothorax apparaissent facilement (2 à $6,4 \%$ des cas) du fait de déficits de la coagulation. Des thoracotomies pour hémostase et décaillotage et/ou des embolisations en salle de radiologie interventionnelle sont alors souvent nécessaires.

Les complications infectieuses, bien que non spécifiques, sont particulièrement fréquentes. La publication australienne et néo-zélandaise sur l'utilisation de l'ECMO pendant l'épidémie de grippe en 2009 rapporte une incidence de $62 \%$ d'infections [19]. La plupart sont des infections ou des surinfections pulmonaires et des septicémies. Une infection débutante n'est pas toujours facile à diagnostiquer chez le patient sous assistance extracorporelle du fait du contrôle de la température par l'échangeur thermique externe et des multiples causes possibles aux anomalies biologiques habituellement observées en cas d'infection (hyperleucocytose, thrombopénie, syndrome inflammatoire). En cas de septicémie, un point d'entrée au niveau des canules doit être recherché systématiquement, et les canules doivent être changées s'il y a une haute suspicion d'infection à ce niveau.

L'insuffisance rénale est également fréquente et, généralement, associée à un mauvais pronostic. L'incidence réelle est difficile à évaluer du fait de l'utilisation de critères différents selon les études mais, de façon assez constante, le recours à des thérapeutiques d'épuration extrarénale survient dans plus de $50 \%$ des cas $[1,23]$. La mortalité des patients traités par ECMO associée à une épuration extrarénale serait significativement plus élevée que celle des patients n'ayant pas nécessité une substitution de la fonction rénale [23]. Il est possible de tirer profit du circuit d'oxygénation extracorporelle pour y brancher un circuit d'ultrafiltration ou de dialyse.

Les principales complications et leurs incidences respectives rapportées dans la littérature sont reprises dans la Tableau 2.

\begin{tabular}{|lc|}
\hline Tableau 2 Principales complications rencontrées et leur fréquence \\
\hline Hémorragies & $\pm 50 \%$ \\
Site de canulation & $17-22 \%$ \\
Gastro-intestinales & $5-10 \%$ \\
Voies respiratoires & $8-13 \%$ \\
Intracrâniennes & $4-12 \%$ \\
Site chirurgical & $16 \%$ \\
Hémolyse & $7-10 \%$ \\
CIVD & $2-4 \%$ \\
Infections & $20-60 \%$ \\
Respiratoires & $44 \%$ \\
Septicémies & $21 \%$ \\
\hline CIVD : coagulation intravasculaire disséminée. \\
\hline
\end{tabular}


Assez souvent, une trachéotomie est réalisée pour faciliter la levée de la sédation et envisager le sevrage de l'ECMO. La trachéotomie peut être percutanée ou chirurgicale en fonction de l'anatomie du patient et de l'expérience des équipes. Les investigateurs australiens et néo-zélandais rapportent la réalisation d'une trachéotomie chez $57 \%$ des patients traités par ECMO pour pneumonie à virus influenza H1N1 [19].

\section{Phase de sevrage}

L'amélioration de la fonction pulmonaire apparaît assez simultanément sur les paramètres de la mécanique ventilatoire (augmentation de la compliance pulmonaire), des échanges gazeux (meilleure oxygénation et rapport $\mathrm{PaO}_{2} /$ $\mathrm{FiO}_{2}$ ) et de la radiographie pulmonaire (éclaircissement des champs pulmonaires). Quand ces signes apparaissent, il est possible de diminuer progressivement le débit de la circulation extracorporelle. Si l'on suit la recommandation d'utiliser le débit le plus bas possible qui permet d'obtenir des valeurs de $\mathrm{PaO}_{2}$ et $\mathrm{P}_{\mathrm{a}} \mathrm{CO}_{2}$ satisfaisantes, ce sevrage se fait assez automatiquement au fur et à mesure de la récupération de la fonction pulmonaire. Lorsque le débit atteint 1 à $2 \mathrm{l} / \mathrm{min}$ (ce qui représente habituellement moins de $30 \%$ de support externe), un essai d'arrêt du support peut être tenté. Les paramètres du respirateur sont ajustés à un niveau acceptable (pression inspiratoire, pression positive de fin d'expiration, fréquence, $\mathrm{FiO}_{2}$ ). Le débit du circuit et l'anticoagulation seront maintenus alors que le balayage est arrêté au niveau de l'oxygénateur membranaire. Si les pressions partielles des gaz sanguins artériels se maintiennent pendant deux heures, le patient est prêt pour le retrait des canules. En cas de doute, il n'y a aucun inconvénient à prolonger l'essai même pour toute une nuit s'il le faut.

Si l'amélioration tarde à venir, un traitement par corticoïde peut être discuté, pour autant que le patient ne soit pas dans une situation septique qui en contre-indiquerait l'usage. La durée moyenne du support est d'une dizaine de jours dans la plupart des séries, mais la récupération peut être plus longue. Le support le plus prolongé suivi de succès rapporté dans la littérature dépasse les 100 jours d'ECMO.

Quand la décision est prise de retirer les canules, l'héparine doit être arrêtée, et les canules sont retirées une heure environ après l'arrêt de l'anticoagulation. Une sédation légère est appliquée, puis les canules sont retirées en prenant soin d'éviter l'entrée d'air par les orifices latéraux des canules veineuses (en demandant une manœuvre de Valsalva ou en administrant une courte curarisation). Une légère pression sera appliquée sur la veine, ou une suture sera placée autour de la canule avant son retrait et serrée au moment de son enlèvement.

\section{Autres possibilités}

Lorsque l'option thérapeutique est de diminuer au maximum l'agressivité de la ventilation mécanique sans rechercher à améliorer l'oxygénation, des techniques d'extraction du $\mathrm{CO}_{2}$ par bas débit extracorporel ont été développées grâce à l'utilisation d'oxygénateurs membranaires offrant peu de résistance à l'écoulement sanguin et étant très efficaces pour l'élimination du $\mathrm{CO}_{2}$. La première technique consiste à utiliser un circuit externe d'assistance sans pompe (pumpless ExtraCorporeal Lung Assist ou pECLA) en créant un circuit artérioveineux et en utilisant le cœur du patient pour créer le débit et sa pression sanguine artérielle comme pression de perfusion du circuit. Il est, dès lors, indispensable que le patient génère un index cardiaque minimal de $3 \mathrm{l} / \mathrm{min}$ par $\mathrm{m}^{2}$ avec une pression artérielle moyenne de $70 \mathrm{mmHg}$. Le flux dans le circuit dépend des paramètres hémodynamiques du patient, de la taille des canules artérielles de drainage et veineuse de retour ainsi que de la résistance de l'oxygénateur. Avec une canule artérielle de 15-17 Fr et une canule veineuse de 17-19 Fr, des débits de l'ordre de 1-2 1/min sont obtenus. Dès la mise en route du circuit, on observe une modeste amélioration de l'oxygénation, mais une normalisation rapide de la $\mathrm{PaCO}_{2}$ permettant de réduire les pressions et la fréquence du respirateur. Dans une série de 159 patients traités pour SDRA ou pneumonie pour une durée moyenne de $7 \pm 6,2$ jours, Flörchinger et al. rapportent la nécessité de changer d'oxygénateur 55 fois, suite à l'apparition de thrombus, la rétention de bulles d'air et de rares fuites plasmatiques. Une thrombose complète du circuit est survenue chez $5 \%$ des patients principalement du fait d'une anticoagulation insuffisante. Une ischémie de membre est apparue dans $8 \%$ des cas nécessitant un changement de dimension de la canule ou de positionnement. Un patient a dû subir une amputation de la jambe [24]. Dans une étude plus récente où le diamètre de la canule artérielle était choisi en fonction du diamètre échographique de l'artère et ne dépassait pas $15 \mathrm{Fr}$, l'incidence de complications ischémiques est tombée à $1 \%$ [8]. La place exacte de cette approche reste cependant largement à préciser.

Une autre possibilité est d'utiliser un circuit veinoveineux avec un cathéter unique à double lumière, une pompe créant des débits de 0,5 à $2 \mathrm{l} / \mathrm{min}$ et un oxygénateur membranaire. Cette technique a montré sa capacité à éliminer une grande partie du $\mathrm{CO}_{2}$ produit et à réduire la ventilation mécanique, mais sa place dans le traitement du SDRA reste à démontrer [25-27].

Conflit d'intérêt : le Dr L. Jacquet est consultant pour Thoratec. 


\section{Références}

1. Paden M, Conrad S, Rycus P, et al (2013) Extracorporeal Life Support Organization Registry Report 2012. ASAIO J 59: 202-10

2. Extracorporeal Life Support Organization (ELSO) General Guidelines for all ECLS cases. http://www.elso.med.umich.edu/WordForms/ELSO\%20Guidelines\%20General\%20All\%20ECLS\%20 Version1.1.pdf (accessed 4 June 2013)

3. Chambers S, Ceccio S, Annich G, Bartlett R (1999) Extreme negative pressure does not cause erythrocyte damage in flowing blood. ASAIO J 45:431-5

4. Brodie D, Bacchetta M (2011) Extracorporeal membrane oxygenation for ARDS in adults. N Engl J Med 365:1905-14

5. Wang D, Zhou X, Liu X, et al (2008) Wang-Zwische double lumen cannula-Toward a percutaneous and ambulatory paracorporeal artificial lung. ASAIO J 54:606-11

6. Javidfar J, Wang D, Zwischenberger J, et al (2011) Insertion of bicaval dual lumen extracorporeal membrane oxygenation catheter with image guidance. ASAIO J 57:203-5

7. Gattinoni L, Kolobow T, Tomlinson T, et al (1978) Low-frequency positive pressure ventilation with extracorporeal carbon dioxyde removal (LFPPV-ECCO2R): an experimental study. Anesth Analg $57: 470-7$

8. Bein T, Weber-Carstens S, Goldmann A, et al (2013) Lower tidal volume strategy $(\sim 3 \mathrm{ml} / \mathrm{kg})$ combined with extracorporeal $\mathrm{CO}_{2}$ removal versus "conventional" protective ventilation $(6 \mathrm{ml} / \mathrm{kg})$ in severe ARDS. The prospective randomized Xtravent-study. Intensive Care Med 39:847-56

9. Shapiro A (1995) Antithrombin deficiency in special clinical syndromes- Part I: Extracorporeal Membrane Oxygenation. Sem Hematol 32:33-6

10. Pollock M, Owings J, Gosselin R (1995) ATIII replacement during infant extracorporeal support. Thromb Haemost 73:936

11. Ichinose K, Okamoto T, Tanimoto H, et al (2004) Comparison of a new heparin-coated dense membrane lung with nonheparin-coated dense membrane lung for prolonged extracorporeal lung assist in goats. Artif Organs 28:993-1001

12. Zimmerman A, Aebert H, Reiz A, et al (2004) Hemocompatibility of PMEA coated oxygenators used for extracorporeal circulation procedures. ASAIO J 50:193-9

13. McLaren G, Combes A, Bartlett R (2012) Contemporary extracorporeal membrane oxygenation for adult respiratory failure: life support in the new era. Intensive Care Med 38:210-20
14. Meha N, Halwick D, Dodson B, et al (2007) Potential drug sequestration during extracorporeal membrane oxygenation: results from an ex-vivo experiment. Intensive Care Med 33:1018-24

15. Preston T, Ratliff T, Gomez D, et al (2010) Modified surface coatings and their effect on drug adsorption within the extracorporeal life support circuit. J Extra Corpor Technol 42:199-202

16. Heilmann C, Geisen U, Beyersdorf F, et al (2012) Acquired von Willebrand syndrome in patients with extracorporeal life support (ECLS). Intensive Care Med 38:62-8

17. Bartlett R, Gattinoni L (2010) Current status of extracorporeal life support (ECMO) for cardiopulmonary failure. Minerva Anestesol 76:534-40

18. Noah M, Peek G, Finney S, et al (2011) Referral to an extracorporeal membrane oxygenation center and mortality among patients with severe 2009 influenza A(H1N1) JAMA 306:1659-68

19. The Australia and New Zealand extracorporeal membrane oxygenation (ANZ ECMO) influenza investigators (2009) Extracorporeal membrane oxygenation for 2009 influenza A(H1N1) acute respiratory distress syndrome. JAMA 302:1888-95

20. Mejak B, Giacomuzzi C, Heller E, et al (2004) Argatroban usage for anticoagulation for ECMO on a post-cardiac patient with heparin-induced thrombocytopenia. J Extra Corpor Technol 36:178-81

21. Pappalardo F, Maj G, Scandroglio A, et al (2009) Bioline heparin-coated ECMO with bivalirudine anticoagulation in a patient with acute heparin-induced thrombocytopenia: the immune reaction appeared to continue. Perfusion 24:135-7

22. Koster A, Huebler S, Potapov E, et al (2007) Impact of heparin-induced thrombocytopenia on outcome in patients with ventricular assist device support: a single-institution experience in 358 consecutive patients. Ann Thorac Surg 83:72-6

23. Kielstein J, Heiden A, Beutel G, et al (2013) Renal function and survival in 200 patients undergoing ECMO therapy. Nephrol Dial Transplant 28:86-90

24. Flörchinger B, Philipp A, Klose A, et al (2008) Pumpless extracorporeal lung assist: a 10-year institutional experience. Ann Thorac Surg 86:410-7

25. Burki N, Mani R, Herth F, et al (2013) A novel extracorporeal $\mathrm{CO}_{2}$ removal system. Results of a pilot study of hypercapnic respiratory failure in patients with COPD. Chest 143:678-86

26. Batchinsky A, Jordan B, Regn D, et al (2011) Respiratory dialysis: reduction in dependence on mechanical ventilation by venovenous extracorporeal $\mathrm{CO}_{2}$ removal. Crit Care Med 39:1382-7

27. Sanchez-Lorente D, Jungebluth P, Rovira I, et al (2010) Single double-lumen venous-venous pump-driven extracorporeal lung membrane support. J Thorac Cardiovasc Surg 140:558-63 